\title{
Link between scrapie and BSE?
}

SIR-Peter Aldhous recently reported ${ }^{1}$ the view that transplacental passage of scrapie infectivity occurs in scrapieinfected ewes. But there are contradictory reports concerning this issue, reflecting our ignorance about the origins, spread and pathogenesis of natural scrapie. The elusive aetiology of scrapie underscores the difficulties encountered in designing and interpreting studies. As we describe here, some view natural scrapie as a virallike illness whereas others describe it as a genetic disorder that happens to be experimentally transmissible.

Over the past 5 years, molecular biological and genetic data have profoundly altered our views of experimental scrapie in rodents and the trinity of human prion diseases - kuru, Creutzfeldt-Jakob disease (CJD) and Gerstmann-Sträussler syndrome (GSS). Indeed, the application of modern techniques to this enigmatic area of biology has begun to provide explanations about how a disease can be both genetic and experimentally or iatrogenically infectious ${ }^{2.3}$. The main, and possibly only, component of the transmissible particle (or prion) in scrapie is an abnormal isoform of the host-encoded prion protein $(\operatorname{PrP})^{4}$, which is generated by an as yet undefined post-translational process. That PrP has a central role in scrapie transmission and pathogenesis is independently supported by studies on the purification of scrapie prions, on the molecular genetics of scrapie incubation time genes and by analyses of infected neuroblastoma cells ${ }^{3}$.

Maternal (and lateral) contagious transmission of natural scrapie was first suggested by crosses of scrapied and scrapiefree Suffolk sheep. Progeny of affected ewes rather than rams were about seven times more likely to develop the disease $\mathrm{e}^{5}$. In a subsequent study of Suffolks crossed with Scottish Blackface sheep a similar but reduced tendency was again apparent; ratios of approximately 1.9 and 1.3 to 1 were obtained in experiments involving 54 and 66 offspring, respectively. A background' scrapie incidence of up to 50 per cent in the progeny of unaffected parents, interpreted in terms of lateral contagious transmission, was apparent in this later study ${ }^{6}$. A tendency favouring maternal transmission in Parry's data ${ }^{7}$ on twins born of scrapie-infected ewes was noted by

1. Aldhous, P. Nature 344, 183 (1990)

2. Hsiao, K. et al. Nature 338, 342-345 (1989).

3. Prusiner, S. B. A. Rev. Microbiol. 43, 345-374 (1989).

4. Oesch, B. et al. Cell 40, 735-746 (1985).

5. Oesch, B. et al. Cell 40, 735-746 (1985).

Dickinson, A.G., Young, G.B., Stamp,
C.C. Heredity 20, 485-503 (1965).

6. Dickinson, A.G., Stamp, J.T. \& Renwick, C.C. J. comp. Path. 84, 19-25 (1974).

7. Parry, H.B. Heredity 17, 75-105 (1962).

8. Hadlow, W.J. Race, R.E. Kennedy, R.C. \& Eklund, C.M. in Slow Transmissible Diseases of the Nervous System Vol. 2 (eds Prusiner, S.B. \& Hadlow, W.J.) 3-12 (Academic, New York 1979)
Dickinson et al. ${ }^{5}$, although the numbers quoted for the progeny of unaffected ewes crossed with affected rams do not seem to correspond to the original data. But most of Parry's data on Suffolk sheep revealed no overt tendency for ewes rather than rams to transmit the disease ${ }^{7}$. Parry also found no evidence for contagious transmission - scrapie-free animals produced no affected offspring, even when crossed with scrapied animals.

The hypothesis of maternal transmission can be directly tested by bioassay for infectivity in scrapied ewes. Hadlow and coworkers failed to detect infectious titre in the uterus, ovary or mammary gland of clinically affected Suffolk ewes ${ }^{8}$. Two papers ${ }^{9.10}$ have been widely cited as indicating infectivity in the placentae of Swaledale ewes with scrapie. Unfortunately, negative controls for these experiments were not described and the incubation times in the inoculated recipients were scattered; an alternative explanation is that the observed instances of scrapie represent cross-contaminated inocula (but see ref. 15).

A few cases of transmission from experimentally inoculated ewes to offspring been observed ${ }^{11.12}$, but in an embryo transfer study by W. Foote et al. (personal communication) none of the offspring developed the disease within an observation period greater than or equal to 5 years $(n=86)$. Direct inoculation of control animals in these experiments produced a scrapie incidence of more than 51 per cent. Similarly, negative results were obtained for maternal transmission in experimental scrapie of goats ${ }^{13}$. Early reports suggesting maternal transmission of scrapie in mice have subsequently been strongly challenged. A maternal effect is not apparent in the transmission of either familial or experimental CJD, kuru or GSS.

In summary, the question of maternal transmission represents part of the larger issue of whether natural scrapie in sheep is a contagious disease under normal conditions of husbandry. As bovine spongiform encephalopathy (BSE) is inferred to have arisen by oral-dosing with contaminated feedstuff ${ }^{1+}$, and given that some scrapie researchers considered that natural and experimental scrapie were not necessarily identical diseases ${ }^{.15}$, the data on natural scrapie may be irrelevant to the antici-

. Pattison, I.H. Hoare, M.N. Jebbett, JN \& Watson, W.A. Vet. Rec. 90, 465-468 (1972)

10. Pattison, I.H., Hoare, M.N., Jebbett, J.N. \& Watson, W.A. Br. vet. J. 130, 1xv-1xvii (1974).

11. Gordon, W.S. Report of Scrapie Seminar US Dept. Agric. Publ. ARS 91-53, 19-36 (1964).

12. Dickinson, A.G., Young, G.B. \& Renwick, C.C. Report of Scrapie Seminar. US Dept. Agric. Publ. ARS 91-53. 244-247 (1964)

13. Pattison IH Vet Rec. 76, 333-336 (1964)

14. Wilesmith, J.W. et al. Vet. Rec. 123, 638-644 (1988).

5. Wilesmith, J.W. et al. Vet. Rec. 123, 638-644

16. Scott, M. et al. Cell59, 847-857 (1989). pated behaviour of BSE. But as the dataset on experimental scrapie contains evidence both for and against 'maternal' transmission, and as there is a potential human health hazard concerning BSE, the resolution of this matter must come from future experimentation. Because our studies with transgenic mice ${ }^{\mathrm{it}}$ show that the species barrier for passage of scrapie prions between rodent species is likely to reside in the amino-acid sequence of $\operatorname{PrP}$, similar experiments seem relevant in examining the potential for transmission of prions from beef and sheep products to humans.

D. Westaway S. B. PRUSINER

Departments of Neurology and of

Biochemistry and Biophysics,

University of California,

San Francisco,

California 94143-0518, USA

\section{Frustrated crystallographers}

SIR-Dover's News and Views article', indicating that "many frustrated crystallographers are left wondering why their crystals will not grow large enough or wellordered enough, or grew yesterday but will not grow today", brought back memories of my own experiences with this problem. When I first joined Professor Tom Blundell's group, good-sized crystals of the newly discovered avian pancreatic polypeptide hormone (aPP) had just been grown by Dr Steve Wood. But the small pot of crystals was soon depleted by lowresolution data collection and by screening more than 30 heavy-atom soaks by precession photography.

Subsequent laborious preparation of new materials and numerous crystallization trials would no longer result in crystals. Suspecting the presence of an unknown co-factor, I analysed the crystals using scanning electron microscopy. The microscope was fitted with an EDAX system which could potentially be used to identify trace elements by measuring their

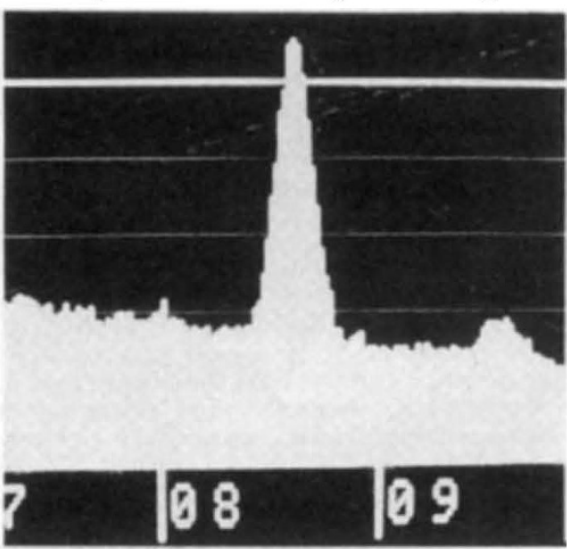

EDAX spectrum from an aPP crystal indicating the presence of zinc. 